\title{
Primary Melanoma of the Small Intestine with a Metastatic Lymph Mass in the Inguinal Canal: A Case Report
}

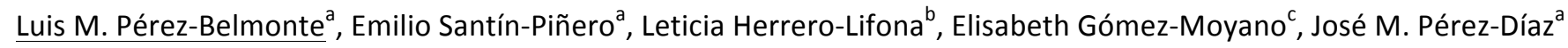

${ }^{a}$ Department of Internal Medicine, Axarquía County Hospital, Vélez-Málaga, Spain

${ }^{b}$ Department of Allergology, University Regional Hospital of Málaga, Málaga, Spain

'Department of Dermatology, University Regional Hospital of Málaga, Málaga, Spain

\begin{abstract}
Objectives: We report a case of primary melanoma of the small intestine. Primary intestinal melanoma (PIM) is an extremely rare neoplasm for which the cause is unknown.
\end{abstract}

Materials and methods: A 67-year-old man was admitted to our department due to abdominal pain, constipation, a large, hard inguinal mass and severe anaemia.

Results: After laboratory data, imaging techniques and histopathological examination, the diagnosis was confirmed. A surgical resection of the intestinal neoplasm, treatment with BRAF inhibitors and radiation therapy to the inguinal mass were performed.

Conclusion: PIM is rare and it is usually difficult to establish its exact origin.

Keywords: Abdominal pain, small intestine, melanoma

Received: 19/09/2014

Accepted: $27 / 09 / 2014$

Published: 03/10/2014

How to cite this article: Pérez-Belmonte LM, Santín-Piñero E, Herrero-Lifona L, Gómez-Moyano E, Pérez-Díaz JM. Primary Melanoma of the Small Intestine with a Metastatic Lymph Mass in the Inguinal Canal: A Case Report, EJCRIM 2014;1:doi: 10.12890/2014_000147

Conflicts of Interests: The authors declare that they have no conflicts of interest related to this research. 


\section{European Journal}

of Case Reports in

Internal Medicine

\section{Introduction}

Intestinal melanomas can be primary tumours or metastases of cutaneous, ocular or anal melanomas. Most melanomas in the small intestine are metastases from primary cutaneous lesions, but a melanoma can also develop as a primary mucosal tumour in the intestinal tract (less than 2\%) [1]. Although it is important to distinguish primary from metastatic disease, establishing the correct origin of small bowel melanoma can be difficult or impossible. In this article, we report a patient presenting with primary melanoma of the small intestine [2].

\section{Case report}

A 67-year-old German man was admitted to the Department of Internal Medicine due to 3 months of intermittent and diffuse abdominal pain, progressive constipation, vomiting, melaena and weight loss (over $20 \mathrm{~kg}$ ). Also, he referred a large $(9 \times 6 \mathrm{~cm})$ and hard inguinal mass on the right side that had gradually grown in the previous 6 weeks (Fig. 1).

No other significant findings were noted at physical examination and his medical history was remarkable only for heavy smoking (60 pack-years) and alcohol abuse (120 g/day).

Laboratory data were within normal limits except for severe iron-deficiency anaemia.

Abdominal ultrasound, barium examination and computerized tomography revealed an infiltrative mass that involved segments of ileal loop with aneurismal dilatation and a large inguinal lymph mass without other pathological lymph nodes (Fig. 2).

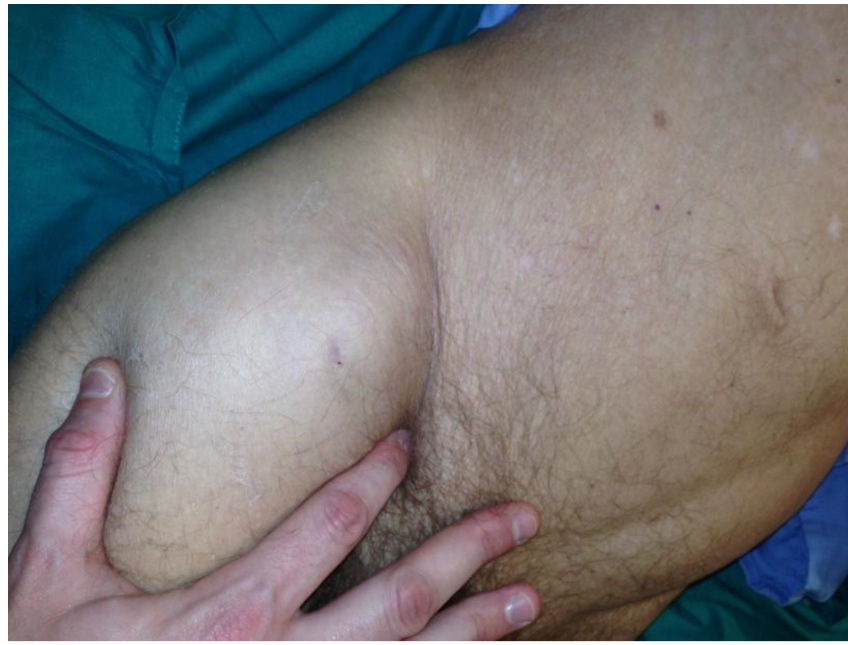

Figure 1: Inguinal mass

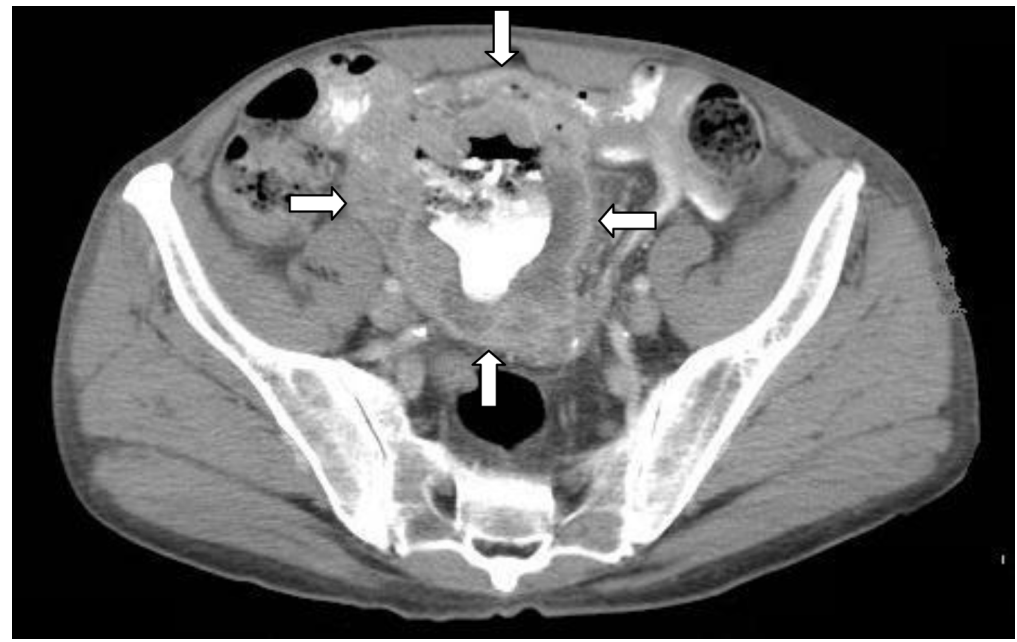

Figure 2: Computer tomography image

A whole positron emission scan confirmed these findings (Fig. 3) and together with total body computerized tomography and urological, ocular and anal examinations ruled out malignancies in other locations.
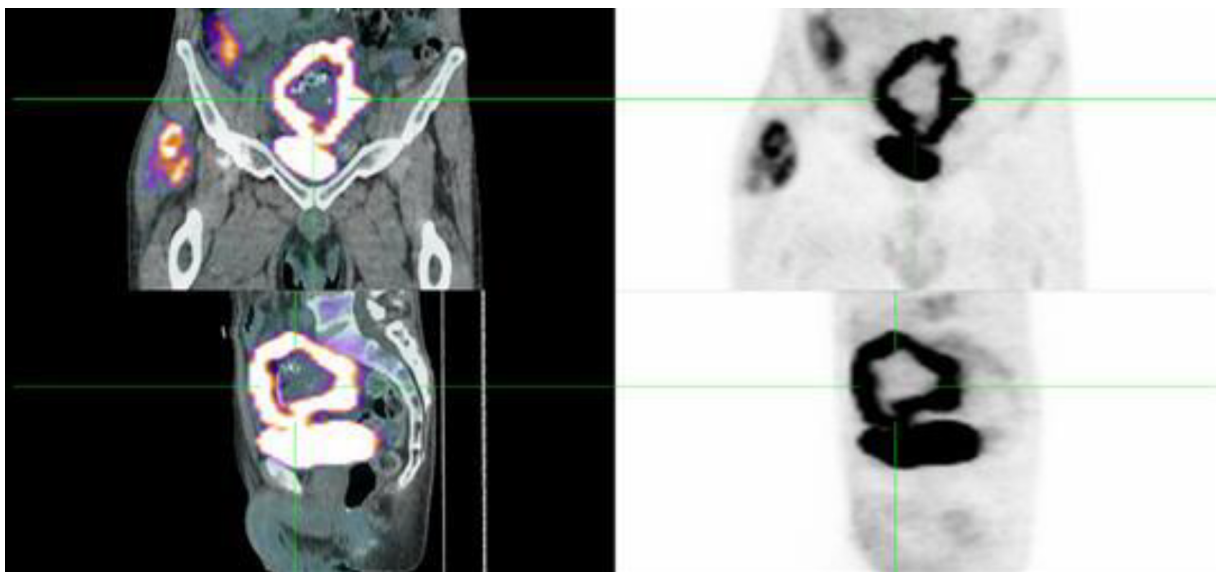

Figure 3: Positron emission scan image 
After several blood transfusions and intensive intravenous iron therapy, a surgical resection of the ileal mass was performed. During laparotomy, an 8-cm mass was identified, involving segments of the ileum. There was no evidence of other abdominal lesions.

The pathological examination of the resected specimen confirmed the diagnosis of melanoma of the small intestine. It showed a precursor lesion in the intestinal mucosa. The BRAF Val600 mutation was positive.

Based on these findings and the patient's clinical presentation, he was given a diagnosis of primary melanoma of the small intestine with a metastatic lymph mass in the inguinal canal.

The postoperative course was uneventful and the clinical evolution was initially favourable, without requiring more blood transfusions or iron supplements. The patient underwent a combination treatment with BRAF inhibitors and radiation therapy to the inguinal mass, improving his physical status and reducing the mass. Four months later, his physical condition gradually worsened and the inguinal mass grew, with several inguinal lymph nodes appearing on the right side. At the present time, the patient is undergoing palliative care.

\section{Discussion}

The vast majority of cases of intestinal melanomas are metastatic lesions, originating from a primary cutaneous lesion or, less frequently, an ocular, urological or rectal lesion. Melanoma can also develop as a primary mucosal neoplasm in the gastrointestinal tract, although it is extremely rare [1]. Establishing the exact origin (primary or secondary) of small bowel melanoma, especially metastatic lesions from an unknown or a regressed primary cutaneous melanoma can be difficult or impossible [3]. In this case, on further extensive dermatological, ophthalmological, urological and rectal examination, we did not have evidence of another primary lesion, giving the diagnosis of primary melanoma of the small intestine with a metastatic lymph mass in the inguinal canal. The histopathological examination also supported this diagnosis. It usually shows a precursor lesion or melanosis in the intestinal mucosa, although histopathological features should not be considered alone [2, 4].

Men are more prone to primary intestinal melanoma (PIM) than women, and the ileum is the most commonly affected area $[1,5]$. In addition, it tends to be more aggressive and is associated with a worse prognosis than cutaneous melanoma. It is a result of aggressive tumour behaviour with fast growth in the rich vascular and lymphatic supply of the intestinal mucosa and late diagnosis [2, 3].

The clinical picture of small bowel melanoma is similar to the clinical presentation of other tumours involving the small intestine. The most common symptoms are abdominal pain, intestinal obstruction, constipation, haematosis, melaena, anaemia, fatigue, weight loss and the presence of a palpable abdominal mass. Substantial lymphadenopathies can also be found.

Diagnosis should be made by a combination of imaging techniques to improve the sensitivity and specificity of the radiological imaging. Transabdominal ultrasonography, conventional barium contrast studies, endoscopy, computer tomography and positron emission scan may be essential for diagnosis of small bowel melanoma. However, the rate of clinical detection is low (only 10\%-20\%) [2, 3].

No standard therapy exists for the treatment of intestinal melanoma, although surgical removal is the treatment of choice in all patients with resectable melanoma $[1,3]$. Wide surgical resection of the tumour with sufficient free margins 
proximally and distally from the lesion, together with a subtended wedge of mesentery to remove regional lymph nodes, is recommended. Treatment of PIM with chemotherapy has very low response rates [1, 2].

In conclusion, melanoma typically develops where melanocytes are found (skin, eyes, meninges and anal region) but can also develop as a primary tumour in the small intestine, although this is very rare. However, the majority of cases are metastases from cutaneous melanoma. This rare case report is interesting because we present a PIM with a large metastatic lymph mass in the inguinal canal, presenting a diagnostic dilemma for us. PIM should be considered in the differential diagnosis of small bowel tumours, which usually remain a challenge for radiologists, pathologists and clinicians.

\section{Learning Points}

- PIM is an extremely rare neoplasm which should be considered in the differential diagnosis of small intestine tumours.

- A clear distinction between PIM and metastatic intestinal melanoma can be difficult.

- A combination of imaging techniques is crucial for its early diagnosis.

\section{References}

1. Patel K, Ward ST, Packer T, Brown S, Marsden J, Thomson M et al. Malignant melanoma of the gastro-intestinal tract: a case series, Int J Surg 2014;12:523-527.

2. Lens M, Bataille V, Krivokapic Z. Melanoma of the small intestine, Lancet Oncol 2009;10:516-521.

3. Schuchter LM, Green R, Fraker D. Primary and metastatic diseases in malignant melanoma of the gastrointestinal tract, Curr Opin Oncol 2000;12:181-185.

4. Blecker D, Abraham S, Furth EE, Kochman ML. Melanoma in the gastrointestinal tract, Am J Gastroenterol 1999;94:3427-3433.

5. Resta G, Anania G, Messina F. Jejuno-jejunal invagination due to intestinal melanoma, World J Gastroenterol 2007;13:310-312. 